\title{
Letter to the editor regarding "Intra-versus extracorporeal anastomosis in laparoscopic right colectomy: a meta-analysis of 3699 patients"
}

\author{
Jianan Chen ${ }^{1}(\mathbb{D}) \cdot$ Zheng Wang $^{1} \cdot$ Qian Liu $^{1}$
}

Accepted: 8 September 2020 / Published online: 21 September 2020

(C) Springer-Verlag GmbH Germany, part of Springer Nature 2020

Dear Editor:

With great interest, we read the recently published article titled "Intra-versus extracorporeal anastomosis in laparoscopic right colectomy: a meta-analysis of 3699 patients" written by Marie et al. [1]. They conducted a meta-analysis with 24 studies to compare the peri- and postoperative outcomes of intracorporeal anastomosis (IA) with extracorporeal anastomosis (EA) in laparoscopic right colectomy.

In the article, the authors demonstrated that the IA was associated with a decrease in parietal abscesses and also allows a decrease of the time to first gas and stools, surgical repair and length of stay, and the two methods were with similar overall complications. They did great works.

However, in our opinion, improper overall $P$ values at the bottom of Figs. 2, 3, and 5 were published (Fig. 2, $p=0.083$; Fig. $3, p=0.296$; Fig. $5, p=0.301)$. The $P$ values in the figures were inconsistent with that in the main text $(p=0.055 ; p=$
$0.006 ; p=0.006$ ). It is important for a correct figure to highlight a desirable result, or a misunderstanding could be made.

\section{Compliance with ethical standards}

Conflict of interest The authors declare that they have no conflict of interest.

\section{Reference}

1. Selvy M, Mattevi C, Slim K, Pezet D, Pereira B, Le Roy B (2020) Intra-versus extracorporeal anastomosis in laparoscopic right colectomy: a meta-analysis of 3699 patients. Int J Color Dis 35: 1673-1680. https://doi.org/10.1007/s00384-020-03675-y

Publisher's note Springer Nature remains neutral with regard to jurisdictional claims in published maps and institutional affiliations.

Jianan Chen and Zheng Wang contributed equally to this work.

Qian Liu

liuqianncc@126.com

\footnotetext{
Department of Colon and Rectal Surgery, National Cancer Center/ National Clinical Research Center for Cancer/Cancer Hospital, Chinese Academy of Medical Sciences and Peking Union Medical College, Beijing 100021, China
} 